LA FACULTAD DE CONTADURÍA TUXPAN DE LA UNIVERSIDAD VERACRUZANA ANTE LOS DESAFÍOS DEL DESARROLLO SOSTENIBLE.

\title{
LA FACULTAD DE CONTADURÍA TUXPAN DE LA UNIVERSIDAD VERACRUZANA ANTE LOS DESAFÍOS DEL DESARROLLO SOSTENIBLE
}

\section{THE TUXPAN FACULTY OF ACCOUNTING OF THE VERACRUZANA UNIVERSITY IN FRONT OF THE CHALLENGES OF SUSTAINABLE DEVELOPMENT}

\author{
Saulo Sinforoso Martínez*, Edalid Álvarez Velázquez**
}

*Doctorado en Ciencias Administrativas y Gestión para el Desarrollo. Profesor de Tiempo Completo de la Facultad de Contaduría. Universidad Veracruzana, Región Poza Rica-Tuxpan. ORCID: http://orcid.org/0000-0001-6961-5546.

**Doctorado en Gestión Ambiental para el Desarrollo. Directora de la Facultad de Contaduría. Universidad Veracruzana, Región Poza Rica - Tuxpan. Email: edalvarez@uv.mx. ORCID: http://orcid.org/0000-00020251-0376.

Dirección para recibir correspondencia: ssinforoso@uv.mx 
LA FACULTAD DE CONTADURÍA TUXPAN DE LA UNIVERSIDAD VERACRUZANA ANTE LOS DESAFÍOS DEL DESARROLLO SOSTENIBLE.

\section{RESUMEN}

OBJETIVO: Identificar las acciones implementadas durante el semestre Agosto 2018- Enero 2019 por la Facultad de Contaduría (FC) Tuxpan de la Universidad Veracruzana (UV) para promover la educación sobre el desarrollo sostenible y el medio ambiente en los académicos, investigadores y estudiantes.

MATERIAL Y MÉTODO: Este estudio se realiza desde un enfoque descriptivo. Se inicia con una revisión teórica sobre las implicaciones del desarrollo sostenible en las Universidades, se continúa con el análisis de estrategias implementadas en algunas instituciones educativas para abordar el tema y se finaliza con el análisis de acciones implementadas en la Facultad sujeta de estudio. La técnica de recolección de información es el análisis documental, con la ficha de registro como instrumento de recolección de datos, las fuentes de información son los informes de actividades 2018-2019 de la Facultad de Contaduría de la UV Tuxpan y el reporte de actividades semestre Agosto 2018- Enero 2019 de la Coordinación de Investigación de la misma institución.

RESULTADOS: La Facultad de Contaduría Tuxpan de la Universidad Veracruzana, ha emprendido diversas acciones que permiten a los estudiantes comprender el tema del desarrollo sostenible y su aplicación en las organizaciones.

CONCLUSIÓN: El Desarrollo Sostenible ha impactado en las áreas del conocimiento e investigación de las Instituciones de Educación Superior, por lo que su necesidad de estudio y abordaje es significativa, pues al contribuir al éxito empresarial es necesario su desarrollo académico en los estudiantes universitarios.

PALABRAS CLAVE: Instituciones de Educación Superior. Desarrollo Sostenible. Educación.

\section{RESUMEN}

OBJECTIVE: To identify the actions implemented during the semester August 2018- January 2019 by the Tuxpan Faculty of Accounting (FA) of the Veracruzana University (VU) to promote education on sustainable development and the environment in academicians, researchers and students. 
LA FACULTAD DE CONTADURÍA TUXPAN DE LA UNIVERSIDAD VERACRUZANA ANTE LOS DESAFÍOS DEL DESARROLLO SOSTENIBLE.

MATERIAL AND METHOD: This study is carried out from a descriptive approach. It begins with a theoretical review on the implications of sustainable development in universities, then, it continues with the analysis of strategies implemented in some educational institutions to address the issue, and finally it ends with the analysis of the actions implemented in the Faculty under study. The information collection technique is the documentary analysis, with the registration form as a data collection instrument, the sources of information are the 2018-2019 activity reports of the Tuxpan Faculty of Accounting of the Veracruzana University and the semester activity report August 2018- January 2019 of the Research Coordination of the same institution.

RESULTS: The Tuxpan Faculty of Accounting of the Veracruzana University has undertaken various actions that allow students to understand the issue of sustainable development and its application in organizations.

CONCLUSION: Sustainable Development has impacted on the areas of knowledge and research of higher education institutions, so their need for study and approach is significant, since contributing to business success requires academic development in the university students.

KEYWORDS: Institutions of Higher Education. Sustainable Development. Education.

\section{INTRODUCCIÓN}

\section{MARCO TEÓRICO}

El Desarrollo Sostenible definido por la Organización de las Naciones Unidas (ONU) con base al informe de la Comisión Mundial sobre el Medio Ambiente y el Desarrollo (1987) es el desarrollo que satisfaga las necesidades del presente sin comprometer la capacidad de las generaciones futuras para satisfacer sus propias necesidades y busca promover la prosperidad y las oportunidades económicas, un mayor bienestar social y la protección del medio ambiente, lo que compromete a la búsqueda de modelos, estrategias y acciones en las organizaciones, pues son ellas las que satisfacen la mayoría de las necesidades sociales.

El impacto generado por el Desarrollo Sostenible en las organizaciones, trae como consecuencia formar Recursos Humanos con saberes teóricos, heurísticos y axiológicos, que le permitan asumir 
LA FACULTAD DE CONTADURÍA TUXPAN DE LA UNIVERSIDAD VERACRUZANA ANTE LOS DESAFÍOS DEL DESARROLLO SOSTENIBLE.

los desafíos marcados por las tendencias internacionales y nacionales sobre el cuidado y conservación de la naturaleza, las alianzas globales, la promoción de una paz, justa e inclusiva, la seguridad de vida próspera y satisfactoria, así como acabar con la pobreza y el hambre, elementos que integran el concepto del Desarrollo Sostenible; para ello, genera un responsabilidad en las Instituciones de Educación Superior (IES) de promover entre sus académicos, docentes y estudiantes las exigencias de la sociedad.

Las IES ante el tema del Desarrollo Sostenible, juegan un papel clave y significativo, pues con base a la Asociación Nacional de Universidades e Instituciones de educación Superior (2018, p. 25) "Las universidades, los centros de investigación y las instituciones tecnológicas y pedagógicas cuentan con un rico capital intelectual para hacer aportaciones significativas en cada uno de los objetivos de la Agenda que contempla, a partir de una visión de futuro ambiciosa y de cambio sobre las tres dimensiones del Desarrollo Sostenible -económica, social y ambiental-", por tal motivo es necesario analizar las estrategias implementadas en las IES para promover el Desarrollo Sostenible entre sus estudiantes, con la finalidad de dar respuesta a la siguiente interrogante:

¿Qué estrategias aplicó la Facultad de Contaduría de la Universidad Veracruzana Tuxpan, durante el periodo Agosto 2018- Enero 2019, para contribuir a la formación de sus estudiantes y académicos en los temas de sostenibilidad?

Como parte de los antecedentes que integran el estado del arte, se describen algunas estrategias implementadas recientemente por algunas IES tanto en un contexto nacional como Internacional:

En Cuba, algunas universidades, en particular en sus programas educativos del área de Negocios, como es la Licenciatura en Contaduría Pública, han integrado en su mapa curricular experiencias educativas vinculadas con el medio ambiente como "Contabilidad Ambiental".

En Colombia, diversas universidades han tomado la decisión de sumar esfuerzo en beneficio del desarrollo sostenible; prueba de ello, los foros realizados donde se analizan las diversas iniciativas, tales como:

En la Universidad Sergio Arboleda se ha venido construyendo desde hace varios años, a través de la migración desde un comité ambiental, pasando a constituir un departamento ambiental y 
LA FACULTAD DE CONTADURÍA TUXPAN DE LA UNIVERSIDAD VERACRUZANA ANTE LOS DESAFÍOS DEL DESARROLLO SOSTENIBLE.

estableciendo en el 2011 un Instituto de Estudios y Servicios Ambientales-IDEASA (Plata y Rivera, 2018).

La Universidad de Medellín, le ha apostado a la articulación entre académicos y estudiantes para abordar el tema del desarrollo sostenible a través de la elaboración de proyectos de investigación constituyendo los "Semilleros de Investigación", grupos de académicos y estudiantes que buscan desarrollar proyectos de esta temática con apoyo de financiamiento de instituciones gubernamentales, "actualmente, se cuenta con un semillero en permacultura en convenio con la fundación Madre Tierra, en el cual se está elaborando una eco Huerta, ejercicio que puede traer grandes beneficios para la institución" (Delgado, 2018. p. 38).

La Universidad Industrial de Santander, aborda el Desarrollo Sostenible a través de sus diferentes grupos de investigación ambiental, tales como: Centro Nacional de Investigaciones para la Agroindustrialización de Especies Vegetales Aromáticas Medicinales Tropicales (CENIVAM), Comité de Ética en Investigación Científica (CEINCI), Centro de Estudios e Investigaciones Ambientales (CEIAM), Plan de Gestión Integral de Residuos (PGIR), entre otros (Arenas, 2018. p. 46).

Con base al Diagnóstico de la Sostenibilidad Ambiental en las Universidades Españolas (2018), en 2004 se crea, en el seno de la Conferencia de Rectores de las Universidades Españolas (CRUE), un grupo de trabajo sobre calidad ambiental y desarrollo sostenible, para el fomento de la actuación por la sostenibilidad en las universidades españolas. "Desde julio de 2008, este grupo pasó a ser Comisión Sectorial de Calidad Ambiental, Desarrollo Sostenible y Prevención de Riesgos en las universidades (CADEP) y en la actualidad esta comisión se denomina CRUESostenibilidad. Dentro de esta comisión de han desarrollado varios grupos de trabajo centrados en objetivos ambientales concretos".

En México, diversas IES, así como asociaciones han tomado cartas en el asunto e implementado diversas acciones como son la realización de foros, congresos, simposios, encuentros, con la finalidad de contribuir al desarrollo sustentable; por tal motivo, se tiene como objetivo general identificar las acciones implementadas durante el semestre Agosto 2018- Enero 2019, por la Facultad de Contaduría Tuxpan de la Universidad Veracruzana, para promover la educación sobre el Desarrollo Sostenible y el medio ambiente en los académicos, investigadores y estudiantes. 
LA FACULTAD DE CONTADURÍA TUXPAN DE LA UNIVERSIDAD VERACRUZANA ANTE LOS DESAFÍOS DEL DESARROLLO SOSTENIBLE.

La Facultad de Contaduría de la Universidad Veracruzana, campus Tuxpan, en su Plan de Desarrollo plasma el compromiso y responsabilidad en la aplicación de diversas estrategias y acciones para contribuir al desarrollo sostenible en sus tres programas educativos del nivel Licenciatura: Contaduría, Gestión y Dirección de Negocios y Sistemas Computacionales Administrativos, lo que da pauta a la identificación de las estrategias implementadas en beneficio de la temática objeto de estudio.

\section{MATERIAL Y MÉTODO}

Esta investigación tiene un alcance descriptivo y se desarrolla bajo el siguiente procedimiento metodológico.

1. Justificación de la importancia de las universidades en el tema del Desarrollo Sostenible.

2. Revisión teórica de las estrategias en beneficio del Desarrollo Sostenible en las IES en el contexto nacional e internacional.

3. Identificación de las estrategias de la Facultad de Contaduría de la Universidad Veracruzana Tuxpan para contribuir a la educación de la comunidad universitaria en temas del Desarrollo Sostenible.

Tabla 1

Técnica e instrumento de recolección de datos para la identificación de acciones implementadas durante el semestre Agosto 2018- Enero 2019

\begin{tabular}{lccc}
\hline $\begin{array}{l}\text { Variables de } \\
\text { estudio }\end{array}$ & Técnica & $\begin{array}{c}\text { Instrumento de } \\
\text { Recolección de } \\
\text { datos }\end{array}$ & Fuentes de información \\
\hline $\begin{array}{l}\text { Acciones } \\
\text { ambiental y } \\
\text { sustentable }\end{array}$ & $\begin{array}{c}\text { Análisis } \\
\text { documental }\end{array}$ & $\begin{array}{c}\text { Ficha de registro de } \\
\text { datos }\end{array}$ & $\begin{array}{c}\text { Informe de actividad FC-UV-Tuxpan } \\
2018-2019 .\end{array}$ \\
& & $\begin{array}{c}\text { Reporte de actividades semestre Agosto } \\
\text { 2018- Enero 2019 Coordinación de } \\
\text { Investigación FC-UV-Tuxpan. }\end{array}$ \\
\hline
\end{tabular}

Fuente: Elaboración propia con datos del informe de actividad FC-UV-Tuxpan 2018-2019 y el reporte de actividades semestre Agosto 2018- Enero 2019. Coordinación de Investigación FC-UV-Tuxpan.

Con base a la tabla 1, la técnica de recolección de información es el análisis documental, con la ficha de registro como instrumento de recolección de datos, las fuentes de información son el 
LA FACULTAD DE CONTADURÍA TUXPAN DE LA UNIVERSIDAD VERACRUZANA ANTE LOS DESAFÍOS DEL DESARROLLO SOSTENIBLE.

informe de actividad FC-UV-Tuxpan 2018 y los reportes de actividades semestre Agosto 2018Enero 2019 de la Coordinación de Investigación de la misma institución.

\section{RESULTADOS}

La Facultad de Contaduría Tuxpan se encuentra ubicada en la carretera a la Barra Norte Kilómetro 7.5, Ejido de la Calzada, 92880 Tuxpan, Veracruz, las acciones realizadas en beneficio del Desarrollo Sostenible (ver tabla 2).

Tabla 2

Acciones de la Facultad de Contaduría de la Universidad Veracruzana Tuxpan para contribuir a la educación en temas del Desarrollo Sostenible durante el periodo Agosto 2018- Enero 2019

\begin{tabular}{ll}
\hline Acciones implementadas & \multicolumn{1}{c}{ Integrantes } \\
\hline Red de Investigación & $\begin{array}{l}\text { Académicos e Investigadores Nacionales e } \\
\text { Internacionales } \\
\text { Académicos y estudiantes } \\
\text { Semillero de Investigación } \\
\text { Proyectos de Investigación }\end{array}$ \\
$\begin{array}{l}\text { Académicos, Investigadores y estudiantes Nacionales } \\
\text { e Internacionales }\end{array}$ \\
Cuerpo Académico & $\begin{array}{l}\text { Académicos } \\
\text { Productos de Investigación }\end{array}$ \\
Académicos, Investigadores y estudiantes Nacionales \\
Rediseño de planes y programas de estudios \\
Oferta de Posgrado & Académicos \\
\hline
\end{tabular}

Fuente: Elaboración propia (2019).

\section{Red de Investigación}

Con fecha 26 de septiembre de 2018, dentro del marco del espacio generado en el Congreso Internacional de Investigación Academia Journals Tuxpan, reunidos en una de las Salas Ejecutivas de Trabajo de un hotel conocido de la Ciudad de Tuxpan de Rodríguez Cano, Veracruz, México, se constituye la Red Latinoamericana de Investigación en Gestión Ambiental y Desarrollo Sustentable en las Organizaciones (RELIGADESO), siendo la presidenta la Dra. Adalid Álvarez Velázquez. 
LA FACULTAD DE CONTADURÍA TUXPAN DE LA UNIVERSIDAD VERACRUZANA ANTE LOS DESAFÍOS DEL DESARROLLO SOSTENIBLE.

Tabla 3

Integrantes e instituciones participantes de RELIGADESO

\begin{tabular}{|c|c|}
\hline Instituciones & Integrantes \\
\hline $\begin{array}{l}\text { Facultad de Contaduría Universidad Veracruzana, } \\
\text { Tuxpan. }\end{array}$ & $\begin{array}{l}\text { Dra. Adalid Álvarez Velázquez } \\
\text { Mtro. Saulo Sinforoso Martínez }\end{array}$ \\
\hline $\begin{array}{l}\text { Facultad de Contaduría y Administración, Universidad } \\
\text { Veracruzana, Xalapa. }\end{array}$ & $\begin{array}{l}\text { Dr. Jerónimo Domingo Ricardez Jiménez } \\
\text { Dra. Natalia Murrieta Martínez } \\
\text { Mtra. Rosa Isela Aguilar Castillo }\end{array}$ \\
\hline $\begin{array}{l}\text { Facultad de Contaduría y Administración, Universidad } \\
\text { Veracruzana, Coatzacoalcos. }\end{array}$ & $\begin{array}{l}\text { Dra. Teodora González Rodríguez } \\
\text { Dra. Ofelia Tapia García }\end{array}$ \\
\hline $\begin{array}{l}\text { Departamento de Negocios. Universidad } \\
\text { Iberoamericana, Puebla. }\end{array}$ & $\begin{array}{l}\text { Dra. Ivonne Tapia Villagómez } \\
\text { Dr. Héctor Manuel Villanueva Lendechy }\end{array}$ \\
\hline Universidad de Guadalajara. & Dr. Rogelio Rivera Fernández \\
\hline Universidad Autónoma del Carmen. & Dra. Nancy Verónica Sánchez Sulú \\
\hline $\begin{array}{l}\text { Universidad de Camagüey "Ignacio Agramonte } \\
\text { Loynaz". }\end{array}$ & Dr. Arístides Pelegrin Mesa \\
\hline Universidad de Montemorelos. & $\begin{array}{l}\text { Dr. Omar Arodi Flores Laguna } \\
\text { Dra. Adriana Ruíz Beltrán. } \\
\text { Dr. Pedro González Urbina }\end{array}$ \\
\hline $\begin{array}{l}\text { Asociación Nacional de Economistas y Contadores de } \\
\text { Cuba, Provincia de Camagüey. }\end{array}$ & Dra. Inés Josefina Torres Mora \\
\hline
\end{tabular}

Fuente: Elaboración propia con base al informe de actividades 2018-2019, de la Facultad de Contaduría de la Universidad Veracruzana Tuxpan (2019).

El objetivo de esta red de investigación, es promover actividades científicas, académicas y de formación de Recursos Humanos vinculados con la Gestión Ambiental para el Desarrollo Sustentable de las Organizaciones en Latinoamérica, teniendo como Línea de Generación y Aplicación del Conocimiento la Gestión Ambiental para el Desarrollo Sustentable de las Organizaciones. Dentro de las actividades a corto plazo, está la elaboración de un proyecto de investigación, aplicado en cada una de las IES participantes, siendo el objeto de estudio, las externalidades ambientales ocasionadas al agua, y a mediano plazo, se busca el registro como Red Temática ante el Consejo Nacional de Ciencia y Tecnología (CONACYT). 
LA FACULTAD DE CONTADURÍA TUXPAN DE LA UNIVERSIDAD VERACRUZANA ANTE LOS DESAFÍOS DEL DESARROLLO SOSTENIBLE.

\section{Semillero de Investigación}

Se constituye el Semillero de Investigación denominado, Contabilidad Ambiental y Desarrollo Sustentable para las Organizaciones (CADSOR), integrado por académicos y estudiantes de los tres programas educativos adscrita a la Facultad de Contaduría Tuxpan, durante el semestre Agosto 2018- Enero 2019, donde se participó en un Congreso Internacional de Contabilidad Ambiental, presentando dos ponencias, la primera vinculada con los incentivos fiscales verdes y la segunda, con la aplicación de la energía solar, en ambas investigaciones se utilizó como campo de aplicación los hoteles del Municipio de Tuxpan de Rodríguez Cano, Veracruz.

Este semillero, a la fecha realiza investigaciones vinculadas a un proyecto macro sobre la determinación de los costos ambientales, productos que serán presentados en congresos internacionales.

\section{Proyecto de Investigación}

Durante el periodo sujeto de estudio la Facultad de Contaduría Tuxpan registra un proyecto de investigación ante el Sistema de Registro y Evaluación de la Investigación (SIREI) de la Universidad Veracruzana, el objetivo general del proyecto es proponer actividades de gestión ambiental en los hoteles de 3, 4 y 5 estrellas establecidos en Tuxpan Veracruz, México y Santa Lucia, Camaguey, Cuba a partir de la determinación del costo sostenible del agua.

La línea de investigación que se fomenta se denomina "la Contabilidad Ambiental para el Desarrollo Sustentable de las Organizaciones", el proyecto es interinstitucional, pues participan académicos y estudiantes de pregrado y posgrado de dos Instituciones de Educación Superior, por México, la Universidad Veracruzana (U.V.) y por Cuba, la Universidad de Camaguey "Ignacio Agramonte Loynaz", para el caso de la U.V., las Facultades involucradas son la de Contaduría Tuxpan y las Facultades de Administración y Contaduría, Campus Xalapa y Coatzacoalcos.

Por parte de la Universidad de Camagüey, la Facultad de Ciencias Económicas y Empresariales, este proyecto de investigación tiene como objetivos específicos estructurar un estado del arte, que abarque los avances y limitaciones de la Contabilidad de Gestión Ambiental en las Organizaciones, incluyendo las aportaciones científicas sobre el costo sostenible, diseñar y aplicar un instrumento de recolección de datos que facilite determinar el costo sostenible del agua en los hoteles de 3, 4 y 5 estrellas establecidos en Tuxpan y Santa Lucía y realizar propuestas y SINFOROSO-MARTÍNEZ S., ÁLVAREZ-VELÁZQUEZ E. 


\section{Original}

LA FACULTAD DE CONTADURÍA TUXPAN DE LA UNIVERSIDAD VERACRUZANA ANTE LOS DESAFÍOS DEL DESARROLLO SOSTENIBLE.

recomendaciones vinculadas con la gestión ambiental a partir del cálculo del costo sostenible del agua en el sector hotelero objeto de estudio.

\section{Cuerpo Académico}

La Facultad de Contaduría Tuxpan cuenta con un Cuerpo Académico denominado "Sistemas de Información: Educativos y de Gestión Económico - Administrativa Basados en la Sustentabilidad", durante el semestre Agosto 2018- Enero 2019 decide modificar su Línea de Generación y Aplicación del Conocimiento para enfocarla al Desarrollo Sostenible de las Organizaciones.

\section{Productos de Investigación}

Contaduría Tuxpan cuenta con diversos productos científicos realizados entre los académicos y estudiantes enfocados al Desarrollo Sostenible de las organizaciones (ver tabla 3).

\section{Tabla 4}

Producción de la Facultad de Contaduría vinculada con el Desarrollo Sostenible en las organizaciones. Periodo Agosto 2018- Enero 2019

\begin{tabular}{lc}
\hline Concepto & Cantidad \\
\hline Libro de Investigación. & 1 \\
Capítulos de Libro en CD. & 2 \\
Artículos en revisión para ser publicados en revistas Scopus. & 2 \\
Artículos Arbitrados. & 2 \\
Memorias en extenso. & 1 \\
Ponencias en Congresos Internacionales. & 3 \\
Conferencias impartidas. & 2 \\
Tesis Dirigidas nivel Licenciatura. & 3 \\
Tesis de Posgrado (en proceso). & 1 \\
\hline
\end{tabular}

Fuente: Elaboración propia con base al informe de actividades 2018-2019, de la Facultad de Contaduría de la Universidad Veracruzana Tuxpan y el reporte de actividades semestre Agosto 2018- Enero 2019 de la Coordinación de Investigación UV-FC- Tuxpan (2019). 
LA FACULTAD DE CONTADURÍA TUXPAN DE LA UNIVERSIDAD VERACRUZANA ANTE LOS DESAFÍOS DEL DESARROLLO SOSTENIBLE.

6. Rediseño de Planes y Programas de Estudios

Docentes de la Facultad de Contaduría Tuxpan, han participado en las actividades vinculadas con el rediseño del Programa Educativo de la Licenciatura en Contaduría, en dicha actividad se han propuesto algunos temas y experiencias educativas vinculadas al Desarrollo Sostenible, algunos ejemplos son: En la Experiencia Educativa de "Contabilidad de Costos I", se agrega el tema de los Costos Ambientales y además, en el mapa curricular se aprueba la Experiencia Educativa de "Contabilidad Ambiental".

\section{Oferta de Posgrado}

En la Facultad de Contaduría Tuxpan, se han sumado esfuerzo por la constitución de una oferta de posgrado enfocado a la Dirección Estratégica Empresarial, teniendo como una de sus líneas de Generación y Aplicación del Conocimiento el Desarrollo Sostenible Empresarial que comprende el diseño de estrategias empresariales buscando un equilibrio entre lo económico, social y ambiental de las empresas para asegurar su continuidad y posicionamiento en el mercado contribuyendo a la satisfacción de las generaciones presentes y futuras, a través de los incentivos fiscales, la innovación tecnológica y procesos productivos y administrativo vinculadas con el Desarrollo Sostenible.

\section{DISCUSIÓN/ CONCLUSIÓN}

Las IES juegan un papel importante para la aplicación del Desarrollo Sostenible en las Organizaciones, siendo necesario la aplicación de diversas estrategias y acciones que promuevan el Desarrollo Sostenible entre los académicos y estudiantes, pues estos últimos son las futuras generaciones que dentro de sus desafíos como profesionistas es buscar el equilibro anhelado por los organismos internacionales.

La Facultad de Contaduría de la Universidad Veracruzana Tuxpan, ha emprendido diversas acciones para promover la educación bajo un marco de Desarrollo Sostenible, ha realizado diversas gestiones que han despertado el interés de los universitarios en relación a esta temática, prueba de ellos es el dinamismo con el que se desarrollan actividades como el Semillero de Investigación. 
LA FACULTAD DE CONTADURÍA TUXPAN DE LA UNIVERSIDAD VERACRUZANA ANTE LOS DESAFÍOS DEL DESARROLLO SOSTENIBLE.

El Desarrollo Sostenible es un tema que se fomenta en las universidades a través de actividades de investigación; sin embargo, es necesario la intervención del Estado a través de Leyes y Políticas Públicas que promuevan a que no sólo las organizaciones de mediana y grande tamaño apliquen estrategias en beneficio de los elementos que constituyen el Desarrollo Sostenible, sino que también es turno de las Micro y Pequeñas empresas implementar iniciativa en beneficio de la sociedad pues temas como el medio ambiente, la pobreza e inclusión es responsabilidad de todos.

Se requiere mayor colaboración entre Estado- Empresa- Universidad, para afrontar los desafíos del Desarrollo Sostenible. Además, es indispensable que el trabajo realizado en las academias sean planteadas al Estado, para que en un ambiente de consenso sea aplicado en las organizaciones tanto públicas como privadas, con la finalidad de buscar un equilibro que permita salvaguardar la integridad de las futuras generaciones.

\section{PROPUESTAS}

Después de analizar las diversas actividades realizadas en la Facultad de Contaduría Tuxpan, se propone la aplicación de los semilleros de investigación en las IES, como un medio para promover el tema del Desarrollo Sostenible entre la comunidad universitaria, asimismo se propone la elaboración de trabajos colaborativos como las Redes de Investigación y por último, se propone la integración de temas vinculadas con el Desarrollo Sostenible en el mapa curricular de los Programas Educativos de Licenciatura y Posgrado.

\section{REFERENCIAS BIBLIOGRÁFICAS}

Arenas, A. (2018). Ponencia para el Foro de Universidades y Sostenibilidad Ambiental, Universidad Industrial de Santander (UIS). En J. Barahona (Ed.), Universidades y sostenibilidad: Experiencias de las instituciones de educación superior en Colombia (pp. 45-48). Bogotá: Digiprint. Recuperado de https://www.iauhesd.net/sites/default/files/documents/universidades_y_sostenibilidad.pdf

Asociación Nacional de Universidades e Instituciones de Educación Superior. (2018). Visión y acción 2030: Propuesta de la ANUIES para renovar la educación superior en México. 
LA FACULTAD DE CONTADURÍA TUXPAN DE LA UNIVERSIDAD VERACRUZANA ANTE LOS DESAFÍOS DEL DESARROLLO SOSTENIBLE.

Recuperado

de

http://www.anuies.mx/media/docs/avisos/pdf/VISION_Y_ACCION_2030.pdf

Delgado, D. (2018). La educación ambiental: proyecciones para la gestión en la universidad de Medellín. En J. Barahona (Ed.), Universidades y sostenibilidad: Experiencias de las instituciones de educación superior en Colombia (pp. 37-39). Bogotá: Digiprint. Recuperado de https://www.iauhesd.net/sites/default/files/documents/universidades_y_sostenibilidad.pdf

Plata, A. y Rivera, D. (2018). El compromiso ambiental en la Universidad Sergio Arboleda, Bogotá. En J. Barahona (Ed.), Universidades y sostenibilidad: Experiencias de las instituciones de educación superior en Colombia (pp. 29-32). Bogotá: Digiprint. Recuperado de https://www.iau-hesd.net/sites/default/files/documents/universidades_y_sostenibilidad.pdf

Universidad Veracruzana. (2008). Plan General de Desarrollo 2025. Recuperado de http://www.uv.mx/transparencia/files/2012/10/PlanGeneraldeDesarrollo2025.pdf

Universidad Veracruzana. (2018). Informes de actividades 2018-2019: Facultad de Contaduría de la Universidad Veracruzana Poza Rica - Tuxpan. Recuperado de https://www.uv.mx/pozarica/contaduria/files/2019/11/8vo-Informe-2018-2019-

TERMIINADO.pdf

Universidad Veracruzana. (2019). Reporte de Actividades semestre Agosto 2018- Enero 2019. Coordinación de Investigación de la Facultad de Contaduría de la Universidad Veracruzana, Tuxpan.

Universidades Españolas. (2018). Diagnóstico de la sostenibilidad ambiental en las universidades españolas. Recuperado de http://www.crue.org/Boletin_SG/2018/2018.04.10\%20Informe\%20Sostenibilidad\%20Unive rsidades\%20v3.4.pdf 